\title{
An Improved Robust Beamforming Design for Cognitive Multiantenna Relay Networks
}

\author{
Lulu Zhao, ${ }^{1,2}$ Guang Liang, ${ }^{2}$ and Huijie Liu ${ }^{2}$ \\ ${ }^{1}$ Shanghai Institute of Microsystem and Information Technology, Chinese Academy of Sciences, Shanghai 200050, China \\ ${ }^{2}$ Shanghai Engineering Center for Microsatellites, Shanghai 201210, China \\ Correspondence should be addressed to Huijie Liu; hjliu72@hit.edu.cn
}

Received 24 October 2016; Accepted 17 January 2017; Published 14 February 2017

Academic Editor: Alessandro Bazzi

Copyright (C) 2017 Lulu Zhao et al. This is an open access article distributed under the Creative Commons Attribution License, which permits unrestricted use, distribution, and reproduction in any medium, provided the original work is properly cited.

\begin{abstract}
This paper investigates the robust relay beamforming design for the multiantenna nonregenerative cognitive relay networks (CRNs). Firstly, it is proved that the optimal beamforming matrix could be simplified as the product of a variable vector and the conjugate transposition of a known channel response vector. Then, by exploiting the optimal beamforming matrix with simplified structure, an improved robust beamforming design is proposed. Analysis and simulation results show that, compared with the existing suboptimal scheme, the proposed method can achieve higher worst-case channel capacity with lower computational complexity.
\end{abstract}

\section{Introduction}

Cognitive radio [1] has been proposed as a potential technology to improve the spectrum utilization and alleviate the spectrum shortage problem in wireless communication system. In underlay cognitive radio network, the secondary users (SUs) are allowed to share the spectrum licensed to the primary users (PUs) only when the interference power from SUs to PUs remains below a predefined threshold. The threshold is defined as maximum tolerable interference level below which the PUs could maintain reliable communication $[2,3]$. On the other hand, the interference constraint would require very low transmit power for SUs [3], which restricts the achievable channel capacity for SUs. In order to increase the channel capacity, the cognitive relay networks (CRNs) have been actively investigated to help improve the communication of SUs [4-6]. The relaying technique is proved to be an effective way to extend the coverage and enhance the performance of wireless communication systems [7]. Generally, there are mainly two schemes to implement relaying: regenerative scheme and nonregenerative scheme $[8,9]$. In regenerative relaying scheme, the relay decodes the received signal and then retransmits it to the destination receiver after appropriate reencoding, whereas, in nonregenerative relaying scheme, the relay simply scales the received signal and forwards it to the destination receiver. Compared with regenerative scheme, the nonregenerative relaying scheme is relatively simple and more attractive. In addition, beamforming is an efficient and popular approach to exploit the spatial diversity offered by multiple antennas, which has been commonly reported for nonregenerative CRNs with single multiantenna relay $[10,11]$ or multiple relays $[12,13]$.

Most of the beamforming designs mentioned above assume the availability of perfect channel state information (CSI). However, the practical available CSI would be imperfect due to various factors, for example, estimation and feedback errors. In CRNs, more CSI errors may be caused due to the limited cooperation between PUs and SUs. The performance of relay beamforming design, which is based on the assumption of perfect CSI, would degrade in the case with CSI errors. Hence, it is of critical importance to develop robust relay beamforming designs which take into consideration the CSI errors to guarantee the quality of service (QoS) requirement. Robust relay beamforming designs for CRNs have been studied in [13-16]. According to the way that the CSI errors are modeled, the design approaches are mainly divided into chance constrained methods [14, $15]$ and the worst-case constrained methods $[13,16]$. The method of chance constrained robust design assumes that 
the CSI errors are random variables following the known statistical distribution, and the robustness can be achieved in a probabilistic sense; for example, the QoS requirements are met with a high probability, while the method of worstcase constrained robust design assumes that the CSI errors lie in bounded uncertainty regions, and the QoS requirements are guaranteed for all possible errors within the uncertainty regions, thereby, achieving absolute robustness. Based on the latter method, [17] studied the robust beamforming design problem for the multiantenna nonregenerative CRN where a pair of SUs communicate through a multiantenna relay with imperfect CSIs. The SUs and relay share the spectrum licensed to $M$ PUs. The CSI errors are bounded by elliptically uncertainty regions. The objective of the robust beamforming design is to maximize the worst-case achievable channel capacity for SUs by optimizing the relay beamforming matrix, subject to the transmit power constraint of relay and the worst-case interference constraints of PUs. However, the original optimization problem of the robust beamforming design is nonconvex and thus difficult to be solved. Then, as an alternative solution to the original problem, an approximation problem was solved by neglecting the dependence existed between the received signal component and noise component at SU due to the same CSI error. However, because the approximation problem is not equivalent to the original one, the existing method is a suboptimal scheme which often yields suboptimal solutions for the original problem.

In order to avoid the performance loss due to suboptimal solutions, an improved robust relay beamforming scheme for $\mathrm{CRN}$ is proposed in this paper. Firstly, it has been proved that the optimal beamforming matrix could be simplified as the product of a variable vector and the conjugate transposition of a known channel response vector. Then, by utilizing optimal beamforming matrix with simplified structure, the original problem is converted into an equivalent problem instead of the approximation problem solved in [17]. An optimal or near-optimal solution of the original problem can be obtained by solving the equivalent problem. It is proved that the performance loss of the near-optimal solution could be negligible. Therefore, the proposed method can obtain better performance than the suboptimal scheme. In addition, the computational complexity of proposed method is much lower than that of the suboptimal scheme. This is mainly because the variable in the equivalent problem in an $\mathrm{N}$-dimension vector by assuming the number of antennas in the relay is $N$, while that in the approximation one is an $\mathrm{N}$-order matrix.

The rest of the paper is organized as follows. In Section 2, the system model of CRN is presented. In Section 3, the improved robust relay beamforming design for CRN is proposed. In Section 4, numerical simulations are presented to illustrate the performance of the proposed method. Finally, the conclusions are drawn in Section 5.

Notations. Vectors are written in lower case boldface letters, while matrices are denoted by upper case boldface letters. $\mathbf{I}_{n}$ is the $n \times n$ identity matrix and $\mathbf{0}$ is a zero vector or matrix. $\mathbb{C}^{N \times M}$ denotes the space of $N \times M$ matrix with complex entries. The superscript $(\cdot)^{H}$ stands for the Hermitian transposition of a

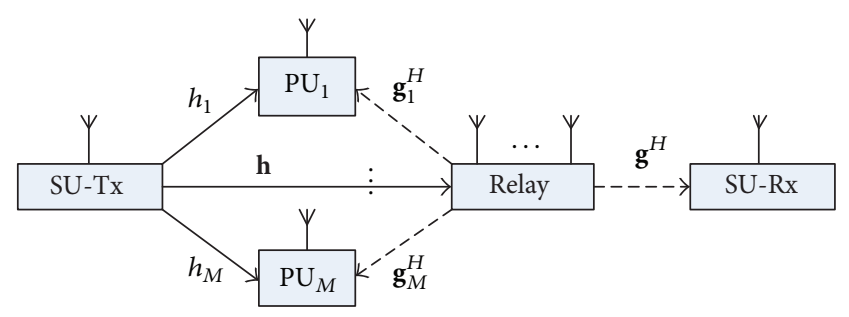

FIgURE 1: The system model for the CRN.

complex vector or matrix. $|\cdot|$ and $\|\cdot\|$ denote the absolute value of a complex scalar and Frobenius norm of a vector or matrix, respectively. $\operatorname{tr}(\mathbf{X})$ and $\operatorname{rank}(\mathbf{X})$ represent the trace and rank of matrix $\mathbf{X}$, respectively. Furthermore, $\mathbf{X} \geq \mathbf{0}$ and $\mathbf{X}>\mathbf{0}$ mean $\mathbf{X}$ is Hermitian positive semidefinite and positive definite matrix, respectively. $\mathbf{x} \sim \mathscr{C} \mathcal{N}(\boldsymbol{\mu}, \boldsymbol{\Sigma})$ represents that the random vector $\mathbf{x}$ follows the circular symmetric complex Gaussian distribution with mean vector $\boldsymbol{\mu}$ and covariance matrix $\Sigma$.

\section{System Model and Problem Formulation}

2.1. System Model. A two-hop nonregenerative CRN is considered which consists of an SU-transmitter (SU-Tx), an SU receiver (SU-Rx), a cognitive relay, and $M$ PUs. The SUs and the relay are allowed to share the same spectrum with $M$ PUs. The relay is equipped with $N$ antennas while other nodes are equipped with single antenna. The same assumption as in [17] applies for the system model; that is, reliable communication link is established by the relay with no direct link between the SU-Tx and SU-Rx. The scenario is typical for relay-assisted device-to-device (D2D) communications where two D2D users in an underlay cellular network communicate with the help of a femtocell $[18,19]$. The configuration is illustrated in Figure 1.

The CRN operates in a half-duplex mode and the communication based on relay takes two time slots. In the first time slot, the SU-Tx transmits signal to the relay. The signal received at the relay can be expressed as

$$
\mathbf{r}=\mathbf{h} s+\mathbf{n}_{r},
$$

where $\mathbf{h}$ denotes the channel response from the SU-Tx to relay, $s$ is the transmit symbol at the SU-Tx with $E\left[|s|^{2}\right]=\sigma_{s}^{2}$, and $\mathbf{n}_{r}$ is the additive Gaussian noise vector at the relay with $\mathbf{n}_{r} \sim \mathscr{C} \mathcal{N}\left(\mathbf{0}, \sigma_{r}^{2} \mathbf{I}\right)$.

In the second time slot, the relay multiplies the received signal $\mathbf{r}$ with a beamforming matrix $\mathbf{R} \in \mathbb{C}^{N \times N}$ and forwards the processed signal to SU-Rx. The signal forwarded by the relay is

$$
\mathbf{x}=\mathbf{R r}=\mathbf{R h} s+\mathbf{R} \mathbf{n}_{r} .
$$

Then, the transmit power of the relay is

$$
\sigma_{s}^{2}\|\mathbf{R} \mathbf{h}\|^{2}+\sigma_{r}^{2}\|\mathbf{R}\|^{2} .
$$

The received signal at the SU-Rx is expressed as

$$
y=\mathbf{g}^{H} \mathbf{x}+n_{d}=\mathbf{g}^{H} \mathbf{R} \mathbf{h} s+\mathbf{g}^{H} \mathbf{R} \mathbf{n}_{r}+n_{d},
$$


where $\mathbf{g}^{H}$ denotes the channel response from the relay to SU$\mathrm{Rx}$ and $n_{d}$ is the additive Gaussian noise at the SU-Rx with $n_{d} \sim \mathscr{C} \mathscr{N}\left(0, \sigma_{d}^{2}\right)$. Then, the received signal-to-noise ratio (SNR) at the SU-Rx can be expressed as

$$
\mathrm{SNR}=\frac{\sigma_{s}^{2}\left|\mathbf{g}^{H} \mathbf{R h}\right|^{2}}{\sigma_{r}^{2}\left\|\mathbf{g}^{H} \mathbf{R}\right\|^{2}+\sigma_{d}^{2}} .
$$

The interference power from the relay to the $m$ th PU can be expressed as

$$
\sigma_{s}^{2}\left|\mathbf{g}_{m}^{H} \mathbf{R h}\right|^{2}+\sigma_{r}^{2}\left\|\mathbf{g}_{m}^{H} \mathbf{R}\right\|^{2},
$$

where $\mathbf{g}_{m}^{H}$ denotes the channel response from the relay to $m$ th PU.

2.2. CSI Errors. As in [17], it is assumed that the practical available CSIs of relay-to-PU links and relay-to-SU-Rx link at relay are imperfect and the actual CSI is within the neighborhood of the imperfect CSI which is obtained from estimation (for relay-to-PU CSI) or feedback information (for relay-to-SU-Rx CSI). Specifically, the actual CSIs $\mathbf{g}$ and $\mathbf{g}_{m}$ can be represented as

$$
\begin{aligned}
\mathbf{g} & =\widehat{\mathbf{g}}+\Delta \mathbf{g}, \\
\mathbf{g}_{m} & =\widehat{\mathbf{g}}_{m}+\Delta \mathbf{g}_{m}, \quad m=1,2, \ldots, M,
\end{aligned}
$$

where $\widehat{\mathbf{g}}$ and $\widehat{\mathbf{g}}_{m}$ denote the imperfect practical available CSIs, respectively, and $\Delta \mathbf{g}$ and $\Delta \mathbf{g}_{m}$ denote the CSI errors for $\mathbf{g}$ and $\mathbf{g}_{m}$, respectively. The CSI errors $\Delta \mathbf{g}$ and $\Delta \mathbf{g}_{m}$ are bounded by the ellipsoidal uncertainty regions

$$
\begin{aligned}
\mathscr{G} & =\left\{\Delta \mathbf{g} \mid \Delta \mathbf{g}^{H} \mathbf{Q} \Delta \mathbf{g} \leq 1\right\}, \\
\mathscr{G}_{m} & =\left\{\Delta \mathbf{g}_{m} \mid \Delta \mathbf{g}_{m}^{H} \mathbf{Q}_{m} \Delta \mathbf{g}_{m} \leq 1\right\}, \quad m=1,2, \ldots, M,
\end{aligned}
$$

respectively, where the matrices $\mathbf{Q}>\mathbf{0}$ and $\left\{\mathbf{Q}_{m}>\mathbf{0}\right\}_{m=1}^{M}$ are used to determine the qualities of CSIs and assumed to be known [17, 20, 21].

2.3. Problem Formulation. The objective of the robust relay beamforming design is to maximize the worst-case achievable channel capacity for SUs by optimizing relay beamforming matrix, subject to worst-case interference constraints at PUs and transmit power constraint at relay. The robust beamforming problem can be expressed as

$$
\begin{array}{cl}
\mathscr{P} 1: \max _{\mathbf{R}} & \min _{\Delta \mathbf{g} \in \mathscr{G}} \frac{1}{2} \log _{2}\left(1+\frac{\sigma_{s}^{2}\left|\mathbf{g}^{H} \mathbf{R} \mathbf{h}\right|^{2}}{\sigma_{r}^{2}\left\|\mathbf{g}^{H} \mathbf{R}\right\|^{2}+\sigma_{d}^{2}}\right) \\
\text { s.t. } \quad & \sigma_{s}^{2}\|\mathbf{R} \mathbf{h}\|^{2}+\sigma_{r}^{2}\|\mathbf{R}\|^{2} \leq P_{r}, \\
& \sigma_{s}^{2}\left|\mathbf{g}_{m}^{H} \mathbf{R h}\right|^{2}+\sigma_{r}^{2}\left\|\mathbf{g}_{m}^{H} \mathbf{R}\right\|^{2} \leq I_{m}, \\
& \forall \Delta \mathbf{g}_{m} \in \mathscr{G}_{m}, \quad m=1,2, \ldots, M,
\end{array}
$$

where $P_{r}$ is the transmit power budget at the relay. Constraint (10c) shows that, to ensure the communication of PUs, the interference power from relay to $m$ th PU should be below a threshold, denoted as $I_{m}$. It is noted that only the relay beamforming optimization is considered in problem $\mathscr{P} 1$.

2.4. Optimal Transmit Power of SU-Tx. In CRN, the interference power from SU-Tx to $m$ th PU should also be below $I_{m}$. Therefore, the transmit power of SU-Tx is limited by

$$
\sigma_{s}^{2}\left|h_{m}\right|^{2} \leq I_{m}, \quad m=1,2, \ldots, M,
$$

where $h_{m}$ denotes the actual channel response from SU-Tx to the $m$ th PU. It is noted that the SU-Tx also has imperfect SU-Tx-to-PU CSI. Then, the actual CSI $h_{m}$ can be expressed as

$$
h_{m}=\widehat{h}_{m}+\Delta h_{m}
$$

where $\widehat{h}_{m}$ denotes imperfect practical available CSI and $\Delta h_{m}$ is the CSI error bounded by $\left|\Delta h_{m}\right|^{2} \leq \epsilon_{m}$. For the imperfect CSI case, constraint (11) should be rewritten as

$$
\max _{\left|\Delta h_{m}\right|^{2} \leq \epsilon_{m}} \sigma_{s}^{2}\left|\widehat{h}_{m}+\Delta h_{m}\right|^{2} \leq I_{m}, \quad \forall m
$$

From (13), we can get

$$
\sigma_{s}^{2} \leq \min \left(P_{s}, \frac{I_{m}}{\left(\left|\widehat{h}_{m}\right|+\sqrt{\epsilon_{m}}\right)^{2}}, \forall m\right),
$$

where $P_{s}$ denotes the transmit power budget of SU-Tx.

Define $\bar{\sigma}_{s}^{2} \triangleq \min \left(P_{s}, I_{m} /\left(\left|\widehat{h}_{m}\right|+\sqrt{\epsilon_{m}}\right)^{2}, \forall m\right)$. In [17], $\bar{\sigma}_{s}^{2}$ is adopted as the transmit power of SU-Tx without illustrating the reasons for adoption of $\bar{\sigma}_{s}^{2}$ (note that the expression of $\bar{\sigma}_{s}^{2}$ in [17] is given with minor error as $\bar{\sigma}_{s}^{2} \triangleq \min \left(P_{s}, I_{m} /\left(\left|\widehat{h}_{m}\right|^{2}+\right.\right.$ $\left.\left.\epsilon_{m}\right), \forall m\right)$ ). In fact, the optimal $\sigma_{s}^{2}$ can be obtained by solving the following problem:

$$
\begin{array}{cl}
\mathscr{P} 2: \max _{\sigma_{s}^{2}, \mathbf{R}} & \min _{\Delta \mathbf{g} \in \mathscr{G}} \frac{1}{2} \log _{2}\left(1+\frac{\sigma_{s}^{2}\left|\mathbf{g}^{H} \mathbf{R} \mathbf{h}\right|^{2}}{\sigma_{r}^{2}\left\|\mathbf{g}^{H} \mathbf{R}\right\|^{2}+\sigma_{d}^{2}}\right) \\
\text { s.t. } \quad & \sigma_{s}^{2} \leq \bar{\sigma}_{s}^{2}, \\
& \sigma_{s}^{2}\|\mathbf{R} \mathbf{h}\|^{2}+\sigma_{r}^{2}\|\mathbf{R}\|^{2} \leq P_{r}, \\
& \sigma_{s}^{2}\left|\mathbf{g}_{m}^{H} \mathbf{R} \mathbf{h}\right|^{2}+\sigma_{r}^{2}\left\|\mathbf{g}_{m}^{H} \mathbf{R}\right\|^{2} \leq I_{m}, \\
& \forall \Delta \mathbf{g}_{m} \in \mathscr{G}_{m}, \quad m=1,2, \ldots, M .
\end{array}
$$

In problem $\mathscr{P} 2$, the worst-case achievable channel capacity for SUs is maximized by jointly optimizing $\sigma_{s}^{2}$ and $\mathbf{R}$. It can be proved below that $\bar{\sigma}_{s}^{2}$ is just the optimal $\sigma_{s}^{2}$ for this problem.

Proof. Assume that the pair $\left(k \bar{\sigma}_{s}^{2}, \mathbf{R}\right)$ is optimal solution of problem $\mathscr{P} 2$, where $k$ satisfies $0<k \leq 1$. If $0<k<1$, it can be verified that $\left(\bar{\sigma}_{s}^{2}, \sqrt{k} \mathbf{R}\right)$ is also feasible and

$$
\frac{k \bar{\sigma}_{s}^{2}\left|\mathbf{g}^{H} \mathbf{R} \mathbf{h}\right|^{2}}{\sigma_{r}^{2}\left\|\mathbf{g}^{H} \mathbf{R}\right\|^{2}+\sigma_{d}^{2}}<\frac{\bar{\sigma}_{s}^{2}\left|\mathbf{g}^{H} \sqrt{k} \mathbf{R} \mathbf{h}\right|^{2}}{\sigma_{r}^{2}\left\|\mathbf{g}^{H} \sqrt{k} \mathbf{R}\right\|^{2}+\sigma_{d}^{2}}
$$


always holds. Therefore, $\left(\bar{\sigma}_{s}^{2}, \sqrt{k} \mathbf{R}\right)$ is a better solution than $\left(k \bar{\sigma}_{s}^{2}, \mathbf{R}\right)$, which is contradictory with the assumption that $\left(k \bar{\sigma}_{s}^{2}, \mathbf{R}\right)$ is the optimal solution. Therefore, if and only if $k=1$, the pair $\left(k \bar{\sigma}_{s}^{2}, \mathbf{R}\right)$ is optimal; that is, $\bar{\sigma}_{s}^{2}$ is the optimal $\sigma_{s}^{2}$ for problem $\mathscr{P} 2$.

After the optimal $\sigma_{s}^{2}$ is determined, problem $\mathscr{P} 2$ is equivalent to problem $\mathscr{P} 1$ with $\sigma_{s}^{2}=\bar{\sigma}_{s}^{2}$. Therefore, solving the former problem has been converted to solving the latter one with given $\sigma_{s}^{2}$ as in [17].

\section{Robust Relay Beamforming Design For CRN}

Using the monotonicity of the logarithmic function, the optimization problem $\mathscr{P} 1$ can be equivalently expressed as

$$
\begin{aligned}
\mathscr{P} 3: \max _{\mathbf{R}} & \min _{\Delta \mathbf{g} \in \mathscr{G}} \frac{\sigma_{s}^{2}\left|\mathbf{g}^{H} \mathbf{R} \mathbf{h}\right|^{2}}{\sigma_{r}^{2}\left\|\mathbf{g}^{H} \mathbf{R}\right\|^{2}+\sigma_{d}^{2}} \\
\text { s.t. } \quad & \sigma_{s}^{2}\|\mathbf{R} \mathbf{h}\|^{2}+\sigma_{r}^{2}\|\mathbf{R}\|^{2} \leq P_{r}, \\
& \sigma_{s}^{2}\left|\mathbf{g}_{m}^{H} \mathbf{R h}\right|^{2}+\sigma_{r}^{2}\left\|\mathbf{g}_{m}^{H} \mathbf{R}\right\|^{2} \leq I_{m}, \\
& \forall \Delta \mathbf{g}_{m} \in \mathscr{G}_{m}, \quad \forall m .
\end{aligned}
$$

3.1. Suboptimal Scheme. In [17], a suboptimal solution to the problem $\mathscr{P} 3$ is obtained by solving the following approximation problem $\mathscr{P} 4$ :

$$
\begin{array}{cl}
\mathscr{P} 4: \max _{\mathbf{R}} & \frac{\min _{\Delta \mathbf{g} \in \mathscr{G}} \sigma_{s}^{2}\left|\mathbf{g}^{H} \mathbf{R} \mathbf{h}\right|^{2}}{\max _{\Delta \mathbf{g} \in \mathscr{G}} \sigma_{r}^{2}\left\|\mathbf{g}^{H} \mathbf{R}\right\|^{2}+\sigma_{d}^{2}} \\
\text { s.t. } \quad & \sigma_{s}^{2}\|\mathbf{R} \mathbf{h}\|^{2}+\sigma_{r}^{2}\|\mathbf{R}\|^{2} \leq P_{r}, \\
& \sigma_{s}^{2}\left|\mathbf{g}_{m}^{H} \mathbf{R h}\right|^{2}+\sigma_{r}^{2}\left\|\mathbf{g}_{m}^{H} \mathbf{R}\right\|^{2} \leq I_{m}, \\
& \forall \Delta \mathbf{g}_{m} \in \mathscr{G}_{m}, \quad \forall m .
\end{array}
$$

However, the objective function (18a) is only the lower bound of (17a) since

$$
\frac{\min _{\Delta \mathbf{g} \in \mathscr{G}} \sigma_{s}^{2}\left|\mathbf{g}^{H} \mathbf{R} \mathbf{h}\right|^{2}}{\max _{\Delta \mathbf{g} \in \mathscr{G}} \sigma_{r}^{2}\left\|\mathbf{g}^{H} \mathbf{R}\right\|^{2}+\sigma_{d}^{2}} \leq \min _{\Delta \mathbf{g} \in \mathscr{G}} \frac{\sigma_{s}^{2}\left|\mathbf{g}^{H} \mathbf{R} \mathbf{h}\right|^{2}}{\sigma_{r}^{2}\left\|\mathbf{g}^{H} \mathbf{R}\right\|^{2}+\sigma_{d}^{2}}
$$

always holds for any matrix $\mathbf{R}$, which means that problem $\mathscr{P} 4$ is not equivalent to problem $\mathscr{P} 3$. As a result, the existing method is not the optimal scheme for problem $\mathscr{P} 3$.

3.2. Proposed Method. To simplify the beamforming problem $\mathscr{P} 3$, we introduce the following Lemma 1.

Lemma 1. Assume $\mathbf{R}_{o}$ is the optimal solution of problem $\mathscr{P} 3$; decompose $\mathbf{R}_{o}=\mathbf{w h}^{H}+\mathbf{T}\left(\mathbf{h}^{\perp}\right)^{H}$, where $\mathbf{w} \in \mathbb{C}^{N \times 1}, \mathbf{T} \in$ $\mathbb{C}^{N \times(N-1)}$, and $\mathbf{h}^{\perp}$ is the orthonormal basis for the null space of $\mathbf{h}$. Then, $\mathbf{w h}^{H}$ is also the optimal solution of problem $\mathscr{P} 3$.

Proof. See Appendix A.
Lemma 1 indicates that the optimal beamforming matrix $\mathbf{R}$ could be a rank-one matrix $\mathbf{w h}^{H}$ with some $\mathbf{w} \in \mathbb{C}^{N \times 1}$. Then, by replacing $\mathbf{R}$ with $\mathbf{w h}^{H}$, the problem $\mathscr{P} 3$ can be equivalently reformulated as

$$
\begin{aligned}
\mathscr{P} 5: \max _{\mathbf{w}} & \min _{\Delta \mathbf{g} \in \mathscr{G}} \frac{\sigma_{s}^{2}|\mathbf{h}|^{4}\left|\mathbf{g}^{H} \mathbf{w}\right|^{2}}{\sigma_{r}^{2}|\mathbf{h}|^{2}\left|\mathbf{g}^{H} \mathbf{w}\right|^{2}+\sigma_{d}^{2}} \\
\text { s.t. } & \left(\sigma_{s}^{2}|\mathbf{h}|^{4}+\sigma_{r}^{2}|\mathbf{h}|^{2}\right)|\mathbf{w}|^{2} \leq P_{r}, \\
& \left(\sigma_{s}^{2}|\mathbf{h}|^{4}+\sigma_{r}^{2}|\mathbf{h}|^{2}\right)\left|\mathbf{g}_{m}^{H} \mathbf{w}\right|^{2} \leq I_{m}, \\
& \forall \Delta \mathbf{g}_{m} \in \mathscr{G}_{m}, \quad \forall m .
\end{aligned}
$$

Define $u(\mathbf{w}) \triangleq \min _{\Delta \mathbf{g} \in \mathscr{G}}\left(\sigma_{s}^{2}|\mathbf{h}|^{4}\left|\mathbf{g}^{H} \mathbf{w}\right|^{2} /\left(\sigma_{r}^{2}|\mathbf{h}|^{2}\left|\mathbf{g}^{H} \mathbf{w}\right|^{2}+\right.\right.$ $\left.\left.\sigma_{d}^{2}\right)\right)$ and $v(\mathbf{w}) \triangleq \min _{\Delta \mathbf{g} \in \mathscr{G}}\left|\mathbf{g}^{H} \mathbf{w}\right|^{2}$. Since $\sigma_{s}^{2}|\mathbf{h}|^{4}\left|\mathbf{g}^{H} \mathbf{w}\right|^{2} /$ $\left(\sigma_{r}^{2}|\mathbf{h}|^{2}\left|\mathbf{g}^{H} \mathbf{w}\right|^{2}+\sigma_{d}^{2}\right)$ is an increasing function with respect to $\left|\mathbf{g}^{H} \mathbf{w}\right|^{2}, u(\mathbf{w})$ can be represented by $v(\mathbf{w})$ as

$$
u(\mathbf{w})=\frac{\sigma_{s}^{2}|\mathbf{h}|^{4} v(\mathbf{w})}{\sigma_{r}^{2}|\mathbf{h}|^{2} v(\mathbf{w})+\sigma_{d}^{2}} .
$$

Since $u(\mathbf{w})$ is also an increasing function with respect to $v(\mathbf{w})$, an alternative method for maximizing $u(\mathbf{w})$ is to maximize $v(\mathbf{w})$. Then, the optimal solution of problem $\mathscr{P} 5$ can also be obtained by solving the following problem $\mathscr{P} 6$ :

$$
\begin{array}{ll}
\mathscr{P} 6: \max _{\mathbf{w}} & v(\mathbf{w}) \\
\text { s.t. } & \left(\sigma_{s}^{2}|\mathbf{h}|^{4}+\sigma_{r}^{2}|\mathbf{h}|^{2}\right)|\mathbf{w}|^{2} \leq P_{r}, \\
& \left(\sigma_{s}^{2}|\mathbf{h}|^{4}+\sigma_{r}^{2}|\mathbf{h}|^{2}\right)\left|\mathbf{g}_{m}^{H} \mathbf{w}\right|^{2} \leq I_{m}, \\
& \forall \Delta \mathbf{g}_{m} \in \mathscr{G}_{m}, \quad \forall m .
\end{array}
$$

Different from $\mathscr{P} 4$, problem $\mathscr{P} 6$ is equivalent to problem $\mathscr{P} 3$. Define $\mathbf{W} \triangleq \mathbf{w} \mathbf{w}^{H}$ and $\alpha=1 /\left(\sigma_{s}^{2}|\mathbf{h}|^{4}+\sigma_{r}^{2}|\mathbf{h}|^{2}\right)$ and introduce an auxiliary variable $\tau$; the problem $\mathscr{P} 6$ can be equivalently converted into

$$
\begin{array}{ll}
\mathscr{P} 7: \quad \max _{\mathbf{W} \geq \mathbf{0}, \tau} & \tau \\
\text { s.t. } \quad & \operatorname{tr}(\mathbf{W}) \leq \alpha P_{r}, \\
& \mathbf{g}_{m}^{H} \mathbf{W} \mathbf{g}_{m} \leq \alpha I_{m}, \quad \forall \Delta \mathbf{g}_{m} \in \mathscr{G}_{m}, \quad \forall m, \\
& \mathbf{g}^{H} \mathbf{W g} \geq \tau, \quad \forall \Delta \mathbf{g} \in \mathscr{G}, \\
& \operatorname{rank}(\mathbf{W})=1 .
\end{array}
$$

Denote the optimal solution of problem $\mathscr{P} 6$ as $\mathbf{w}_{o}$. Then, the pair $\left(\mathbf{w}_{o} \mathbf{w}_{o}^{H}, v\left(\mathbf{w}_{o}\right)\right)$ is the optimal solution of problem $\mathscr{P} 7$. The problem $\mathscr{P} 7$ has semi-infinite constraints (23c) and (23d), which are intractable. To make the problem $\mathscr{P} 7$ tractable, the $\mathcal{S}$-Procedure [22] is employed to convert the constraints (23c) and (23d) into linear matrix inequalities (LMIs) [23]. 
By applying the $\mathcal{S}$-Procedure, constraint (23c) can be reformulated as

$$
\left(\begin{array}{cc}
-\widehat{\mathbf{g}}_{m}^{H} \mathbf{W} \widehat{\mathbf{g}}_{m}+\alpha I_{m}-s_{m} & -\widehat{\mathbf{g}}_{m}^{H} \mathbf{W} \\
-\mathbf{W} \widehat{\mathbf{g}}_{m} & -\mathbf{W}+s_{m} \mathbf{Q}_{m}
\end{array}\right) \geq \mathbf{0}, \quad \forall m
$$

for some $s_{m} \geq 0$ and constraint (23d) can be reformulated as

$$
\left(\begin{array}{cc}
\widehat{\mathbf{g}}^{H} \mathbf{W} \widehat{\mathbf{g}}-\tau-s_{0} & \widehat{\mathbf{g}}^{H} \mathbf{W} \\
\mathbf{W} \widehat{\mathbf{g}} & \mathbf{W}+s_{0} \mathbf{Q}
\end{array}\right) \geq \mathbf{0}
$$

for some $s_{0} \geq 0$. Using (24)-(25) and relaxing the rank-one constraint (23e), problem $\mathscr{P} 7$ can be converted into a convex semidefinite programming (SDP) problem $\mathscr{P} 8$ as follows:

$$
\begin{aligned}
& \mathscr{P} 8: \max _{\mathbf{W} \geq \mathbf{0}, \tau} \tau \\
& \text { s.t. } \operatorname{tr}(\mathbf{W}) \leq \alpha P_{r} \text {, } \\
& \left(\begin{array}{cc}
-\widehat{\mathbf{g}}_{m}^{H} \mathbf{W} \widehat{\mathbf{g}}_{m}+\alpha I_{m}-s_{m} & -\widehat{\mathbf{g}}_{m}^{H} \mathbf{W} \\
-\mathbf{W} \widehat{\mathbf{g}}_{m} & -\mathbf{W}+s_{m} \mathbf{Q}_{m}
\end{array}\right) \\
& \geq \mathbf{0} \text {, } \\
& s_{m} \geq 0, \quad \forall m, \\
& \left(\begin{array}{cc}
\widehat{\mathbf{g}}^{H} \mathbf{W} \widehat{\mathbf{g}}-\tau-s_{0} & \widehat{\mathbf{g}}^{H} \mathbf{W} \\
\mathbf{W} \widehat{\mathbf{g}} & \mathbf{W}+s_{0} \mathbf{Q}
\end{array}\right) \geq \mathbf{0}, \\
& s_{0} \geq 0 \text {. }
\end{aligned}
$$

The convex SDP problem $\mathscr{P} 8$ can be solved by standard inner point method [23]. Denote the optimal solution of problem $\mathscr{P} 8$ as $\left(\mathbf{W}_{o}, \tau_{o}\right)$ with $\tau_{o}=\min _{\Delta \mathbf{g} \in \mathscr{G}} \mathbf{g}^{H} \mathbf{W}_{o} \mathbf{g}$. It is obviously that $\tau_{o}$ is the upper bound of $v\left(\mathbf{w}_{o}\right)$; that is, $v\left(\mathbf{w}_{o}\right) \leq \tau_{o}$ always holds. Moreover, if $\mathbf{W}_{o}$ is rank-one, the pair $\left(\mathbf{W}_{o}, \tau_{o}\right)$ is also the optimal solution of problem $\mathscr{P} 7$. Then, $\mathbf{w}_{o}$ can be obtained by decomposing $\mathbf{W}_{o}=\mathbf{w}_{o} \mathbf{w}_{o}^{H}$ and $v\left(\mathbf{w}_{o}\right)$ achieves its upper bound; that is, $v\left(\mathbf{w}_{o}\right)=\tau_{o}$. Otherwise, consider the following problem $\mathscr{P} 9$ :

$$
\begin{aligned}
\mathscr{P} 9: \quad \min _{\mathbf{W} \geq \mathbf{0}} & \operatorname{tr}(\mathbf{W}) \\
\text { s.t. } \quad & \mathbf{g}_{m}^{H} \mathbf{W} \mathbf{g}_{m} \leq \alpha I_{m}, \quad \forall \Delta \mathbf{g}_{m} \in \mathscr{G}_{m}, \quad \forall m, \\
& \mathbf{g}^{H} \mathbf{W} \mathbf{g} \geq(1-\delta) \tau_{o}, \quad \forall \Delta \mathbf{g} \in \mathscr{G},
\end{aligned}
$$

where $0<\delta<1$. Note that $\min _{\Delta \mathbf{g} \in \mathscr{G}} \mathbf{g}^{H} \mathbf{W}_{o} \mathbf{g}=\tau_{o}>(1-\delta) \tau_{o}$; therefore, $\mathbf{W}_{o}$ is a feasible solution of problem $\mathscr{P} 9$. Denote the optimal solution of problem $\mathscr{P} 9$ as $\widehat{\mathbf{W}}_{o}$; the objective function value $\operatorname{tr}\left(\widehat{\mathbf{W}}_{o}\right)$ satisfies $\operatorname{tr}\left(\widehat{\mathbf{W}}_{o}\right) \leq \operatorname{tr}\left(\mathbf{W}_{o}\right) \leq \alpha P_{r}$.

By applying the $\mathcal{S}$-Procedure, constraint (27b) can be reformulated as

$$
\left(\begin{array}{cc}
\widehat{\mathbf{g}}^{H} \mathbf{W} \widehat{\mathbf{g}}-(1-\delta) \tau_{o}-s_{0} & \widehat{\mathbf{g}}^{H} \mathbf{W} \\
\mathbf{W} \widehat{\mathbf{g}} & \mathbf{W}+s_{0} \mathbf{Q}
\end{array}\right) \succeq \mathbf{0}
$$

for some $s_{0} \geq 0$. Using (24) and (28), problem $\mathscr{P} 9$ can be equivalently transformed into a convex SDP problem $\mathscr{P} 10$ as

$$
\begin{aligned}
& \mathscr{P} 10: \min _{\mathbf{W} \geq \mathbf{0}} \operatorname{tr}(\mathbf{W}) \\
& \text { s.t. } \\
& \left(\begin{array}{cc}
-\widehat{\mathbf{g}}_{m}^{H} \mathbf{W} \widehat{\mathbf{g}}_{m}+\alpha I_{m}-s_{m} & -\widehat{\mathbf{g}}_{m}^{H} \mathbf{W} \\
-\mathbf{W} \widehat{\mathbf{g}}_{m} & -\mathbf{W}+s_{m} \mathbf{Q}_{m}
\end{array}\right) \\
& \geq \mathbf{0} \text {, } \\
& s_{m} \geq 0, \forall m, \\
& \left(\begin{array}{cc}
\widehat{\mathbf{g}}^{H} \mathbf{W} \widehat{\mathbf{g}}-(1-\delta) \tau_{o}-s_{0} & \widehat{\mathbf{g}}^{H} \mathbf{W} \\
\mathbf{W} \widehat{\mathbf{g}} & \mathbf{W}+s_{0} \mathbf{Q}
\end{array}\right) \\
& \geq \mathbf{0} \text {, } \\
& s_{0} \geq 0 \text {. }
\end{aligned}
$$

The problem $\mathscr{P} 10$ can be also solved by standard inner point method. In addition, a lemma about the optimal solution of problem $\mathscr{P} 10$ is presented as follows.

Lemma 2. When $0<\delta<1$, the optimal solution $\widehat{\mathbf{W}}_{o}$ must be rank-one.

Proof. See Appendix B.

Lemma 2 indicates that $\widehat{\mathbf{W}}_{o}$ is rank-one with any small positive $\delta$. Decompose $\widehat{\mathbf{W}}_{o}=\widehat{\mathbf{w}}_{o} \widehat{\mathbf{w}}_{o}^{H}$; then, $\widehat{\mathbf{w}}_{o}$ is a feasible solution of problem $\mathscr{P} 6$. This is because $\widehat{\mathbf{w}}_{o}$ satisfies

$$
\begin{aligned}
\left|\widehat{\mathbf{w}}_{o}\right|^{2} & =\operatorname{tr}\left(\widehat{\mathbf{W}}_{o}\right) \leq \alpha P_{r}, \\
\left|\mathbf{g}_{m}^{H} \widehat{\mathbf{w}}_{o}\right|^{2} & =\mathbf{g}_{m}^{H} \widehat{\mathbf{W}}_{o} \mathbf{g}_{m} \leq \alpha I_{m}, \quad \forall \Delta \mathbf{g}_{m} \in \mathscr{G}_{m}, \forall m .
\end{aligned}
$$

Then, the objective function value $v\left(\widehat{\mathbf{w}}_{o}\right)$ for problem $\mathscr{P} 6$ satisfies

$$
v\left(\widehat{\mathbf{w}}_{o}\right)=\min _{\Delta \mathbf{g} \in \mathscr{G}}\left|\mathbf{g}^{H} \widehat{\mathbf{w}}_{o}\right|^{2}=\min _{\Delta \mathbf{g} \in \mathscr{G}} \mathbf{g}^{H} \widehat{\mathbf{W}}_{o} \mathbf{g} \geq(1-\delta) \tau_{o} .
$$

Substituting $v\left(\mathbf{w}_{o}\right) \leq \tau_{o}$ and $v\left(\widehat{\mathbf{w}}_{o}\right) \geq(1-\delta) \tau_{o}$ into (21) will yield

$$
\begin{aligned}
& u\left(\mathbf{w}_{o}\right)-u\left(\widehat{\mathbf{w}}_{o}\right) \\
& =\frac{\sigma_{s}^{2}|\mathbf{h}|^{4} v\left(\mathbf{w}_{o}\right)}{\sigma_{r}^{2}|\mathbf{h}|^{2} v\left(\mathbf{w}_{o}\right)+\sigma_{d}^{2}}-\frac{\sigma_{s}^{2}|\mathbf{h}|^{4} v\left(\widehat{\mathbf{w}}_{o}\right)}{\sigma_{r}^{2}|\mathbf{h}|^{2} v\left(\widehat{\mathbf{w}}_{o}\right)+\sigma_{d}^{2}} \\
& \leq \frac{\sigma_{s}^{2}|\mathbf{h}|^{4} \tau_{o}}{\sigma_{r}^{2}|\mathbf{h}|^{2} \tau_{o}+\sigma_{d}^{2}}-\frac{\sigma_{s}^{2}|\mathbf{h}|^{4}(1-\delta) \tau_{o}}{\sigma_{r}^{2}|\mathbf{h}|^{2}(1-\delta) \tau_{o}+\sigma_{d}^{2}} \\
& =\frac{\sigma_{s}^{2}|\mathbf{h}|^{4} \sigma_{d}^{2} \cdot \delta \tau_{o}}{\left(\sigma_{r}^{2}|\mathbf{h}|^{2} \tau_{o}+\sigma_{d}^{2}\right)\left(\sigma_{r}^{2}|\mathbf{h}|^{2}(1-\delta) \tau_{o}+\sigma_{d}^{2}\right)} \\
& <\frac{\sigma_{s}^{2}|\mathbf{h}|^{4} \cdot \delta \tau_{o}}{\sigma_{d}^{2}} .
\end{aligned}
$$


(1) solve the SDP problem $\mathscr{P} 8$ to obtain the optimal solution $\left(\mathbf{W}_{o}, \tau_{o}\right)$;

(2) if $\operatorname{rank}\left(\mathbf{W}_{o}\right)=1$ then

(3) Decompose $\mathbf{W}_{o}=\mathbf{w}_{o} \mathbf{w}_{o}^{H}$;

(4) else

(5) Set $\delta=10^{-6}$, solve the SDP problem $\mathscr{P} 10$ to obtain the optimal solution $\widehat{\mathbf{W}}_{o}$;

(6) Decomposed $\widehat{\mathbf{W}}_{o}=\widehat{\mathbf{w}}_{o} \widehat{\mathbf{w}}_{o}^{H}$, set $\mathbf{w}_{o}=\widehat{\mathbf{w}}_{o}$;

(7) end if

(8) Calculate the optimal/near-optimal beamforming matrix $\mathbf{R}_{o}=\mathbf{w}_{0} \mathbf{h}^{H}$.

Algorithm 1: The proposed algorithm.

That is, the performance gap between $u\left(\mathbf{w}_{o}\right)$ and $u\left(\widehat{\mathbf{w}}_{o}\right)$ is less than $\left(\sigma_{s}^{2}|\mathbf{h}|^{4} \tau_{o} / \sigma_{d}^{2}\right) \delta$. Therefore, by setting $\delta$ to be a positive number small enough, for example, $\delta=10^{-6}$, the performance loss of near-optimal solution $\widehat{\mathbf{w}}_{o}$ would be negligible.

The proposed algorithm for robust beamforming problem $\mathscr{P} 1$ is shown in Algorithm 1.

3.3. Computational Complexity. The computational complexity of Algorithm 1 mainly comes from the computational complexity of the SDP problems $\mathscr{P} 8$ and $\mathscr{P} 10$. From [24], the computational complexity for solving an SDP problem within a tolerance $\epsilon$ is $O\left(\left(m n^{3.5}+m^{2} n^{2.5}+m^{3} n^{0.5}\right) \cdot \log (1 / \epsilon)\right)$, where $n$ is the dimension of the semidefinite cone and $m$ is the number of linear constraints. Thus, the computational complexity for solving the SDP problems $\mathscr{P} 8$ and $\mathscr{P} 10$ is $O\left(\left((M+1) N^{7}+\right.\right.$ $\left.\left.(M+1)^{2} N^{5}+(M+1)^{3} N\right) \cdot \log (1 / \epsilon)\right)$. Note that the computational complexity of suboptimal scheme is $O\left(\left((M+1) N^{14}+\right.\right.$ $\left.\left.(M+1)^{2} N^{10}+(M+1)^{3} N^{2}\right) \cdot \log (1 / \epsilon)\right)[17]$; the computational complexity of our proposed method is much lower than that of the suboptimal scheme, especially at large $N$.

\section{Simulations}

In this section, the performance of proposed method is demonstrated through numerical simulations. The channel vectors are assumed as $\mathbf{h}, \widehat{\mathbf{g}}$, and $\widehat{\mathbf{g}}_{m}^{H}, \forall m \sim \mathscr{C} \mathscr{N}(\mathbf{0}, \mathbf{I})$, and the noise power is assumed as $\sigma_{r}^{2}=\sigma_{d}^{2}=\sigma^{2}$. The uncertainty regions are assumed to be norm-bounded; that is, $\mathbf{Q}=$ $\left(1 / \omega_{s}\right) \mathbf{I}$ and $\mathbf{Q}_{m}=\left(1 / \omega_{p}\right) \mathbf{I}, \forall m$, where $\left(\omega_{s}, \omega_{p}\right)$ determines the quality of the CSIs. The maximum interference power thresholds are assumed as $I_{m}=I, \forall m$. Three different CSI errors $\left(\omega_{s}, \omega_{p}\right)=(0.01,0.01),\left(\omega_{s}, \omega_{p}\right)=(0.01,0.1)$, and $\left(\omega_{s}, \omega_{p}\right)=(0.1,0.1)$ and two different maximum interference power thresholds $I / \sigma^{2}=0 \mathrm{~dB}$ and $I / \sigma^{2}=-10 \mathrm{~dB}$ are considered in the simulations. The number of the antennas in the relay $N$ is assumed to be 4 and the number of PUs $M$ is assumed to be 2. CVX toolbox [25] is used to solve the SDP problems numerically. In all simulations, the worst-case achievable channel capacity for SUs, expressed as

$$
\min _{\Delta \mathbf{g} \in \mathscr{G} 2} \frac{1}{2} \log _{2}\left(1+\frac{\sigma_{s}^{2}\left|\mathbf{g}^{H} \mathbf{R} \mathbf{h}\right|^{2}}{\sigma_{r}^{2}\left\|\mathbf{g}^{H} \mathbf{R}\right\|^{2}+\sigma_{d}^{2}}\right),
$$

is evaluated with 1000 randomly generated CSI errors satisfying the model (8) for each channel realization. The simulation results are averaged over 1000 randomly generated channel realizations.

Figure 2 shows the relationship between average worstcase achievable channel capacity of proposed method (denoted as "Pro" in the legend) and ST transmit power $\left(\sigma_{s}^{2} / \sigma^{2}\right)$ at $P_{r} / \sigma^{2}=10 \mathrm{~dB}$, together with the worst-case capacity of suboptimal scheme (denoted as "Sub"). $I / \sigma^{2}$ is set to be $0 \mathrm{~dB}$ and $-10 \mathrm{~dB}$ in Figures 2(a) and 2(b), respectively. It can be observed from Figure 2 that the channel capacity increases with the increasing of $\sigma_{s}^{2}$ as illustrated in Section 2.4. We can find that the proposed method outperforms the suboptimal scheme for all $\sigma_{s}^{2}$. Specially, it can be seen from Figure 2(a) that when $I / \sigma^{2}=0 \mathrm{~dB}, \sigma_{s}^{2} / \sigma^{2}=0 \mathrm{~dB}$, and $\left(\omega_{s}, \omega_{p}\right)=$ $(0.1,0.1)$, the worst-case capacity of proposed method and the suboptimal scheme is about $0.8 \mathrm{bps} / \mathrm{Hz}$ and $0.53 \mathrm{bps} / \mathrm{Hz}$, respectively; that is, the proposed method improves the worst-case capacity of $50 \%$ compared with the suboptimal scheme.

Denote the average worst-case achievable channel capacity of proposed method and the suboptimal scheme as $\gamma_{\text {Pro }}$ and $\gamma_{\text {Sub }}$, respectively. The capacity improvement, defined as $\gamma_{\text {Pro }}-\gamma_{\text {Sub }}$, is shown in Figure 3 for $P_{r} / \sigma^{2}=10 \mathrm{~dB}$. From Figure 3, we can observe that the capacity improvement becomes smaller as $\sigma_{s}^{2}$ increases. This is because the increase of $\sigma_{s}^{2}$ would cause both of the relay transmit constraint (17b) and interference power constraint (17c) becoming tighter, reducing the feasible region of problem $\mathscr{P} 3$. The reduction of feasible region may make the suboptimal solution tend to approach the optimal one, which makes $\gamma_{\text {Sub }}$ closer to $\gamma_{\text {Pro }}$ and leads to a smaller capacity improvement. Moreover, it is shown that the capacity improvement increases with the increase of $\omega_{s}$. The reason is that as $\omega_{s}$ increases, the difference between the objective function (17a) and its lower bound (18a) becomes larger by neglecting the correlation of $\Delta \mathbf{g}$ in suboptimal scheme, leading to the increase of the capacity improvement. Furthermore, the capacity improvement decreases with the increase of $\omega_{p}$ due to the fact that the increasing of $\omega_{p}$ tightens the interference power constraint $(17 \mathrm{c})$, reducing the feasible region of problem $\mathscr{P} 3$. In addition, we can also see that, as $I$ increases, the capacity improvement becomes larger. This is because the increase of $I$ loosens the interference constraint (17c), leading to an expansion of the feasible region of problem $\mathscr{P} 3$. 


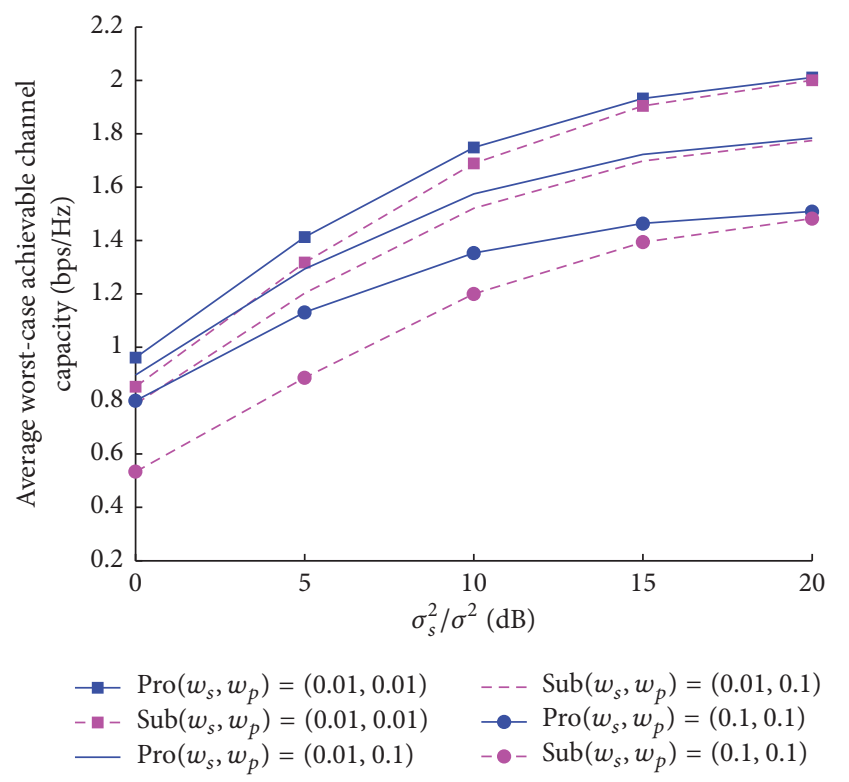

(a)

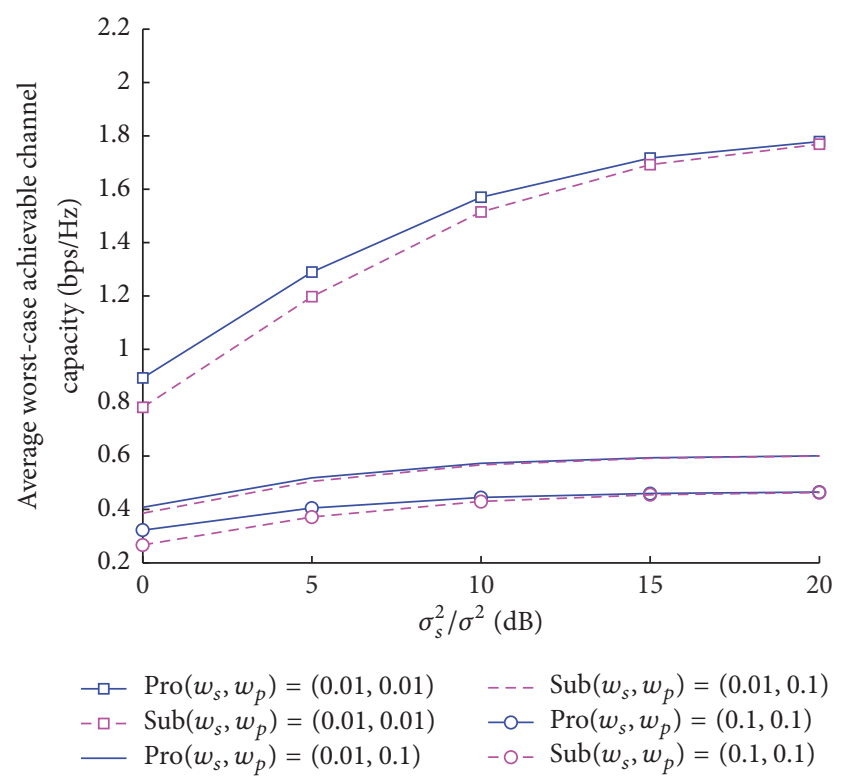

(b)

FIGURE 2: Average worst-case achievable channel capacity versus ST transmit power $\sigma_{s}^{2} / \sigma^{2}$. (a) $I / \sigma^{2}=0 \mathrm{~dB}$; (b) $I / \sigma^{2}=-10 \mathrm{~dB}$.

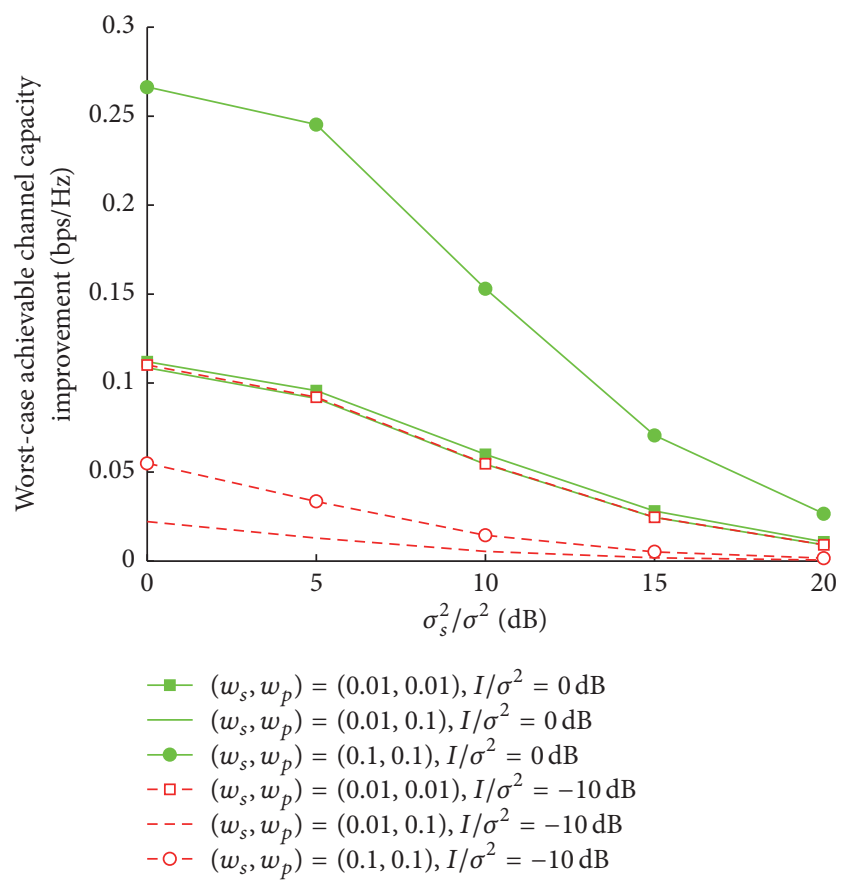

FIGURE 3: Average worst-case achievable channel capacity improvement versus ST transmit power $\sigma_{s}^{2} / \sigma^{2}$.

Figure 4 shows the average worst-case achievable channel capacity versus relay transmit power budget $\left(P_{r} / \sigma^{2}\right)$ at $\sigma_{s}^{2} / \sigma^{2}=5 \mathrm{~dB} . I / \sigma^{2}$ is set to be $0 \mathrm{~dB}$ and $-10 \mathrm{~dB}$ in Figures $4(\mathrm{a})$ and $4(\mathrm{~b})$, respectively. It also can be found from Figure 4 that the proposed method outperforms the suboptimal scheme for all $P_{r}$. The capacity improvement at $\sigma_{s}^{2} / \sigma^{2}=5 \mathrm{~dB}$ is shown in Figure 5. From Figures 4 and 5, we can observe that only when $P_{r}$ is below some threshold, both $\gamma_{\text {Pro }}$ and the capacity improvement increase as $P_{r}$ increases. The reason would be analysis as follows. Consider the following problem:

$$
\begin{array}{cl}
\mathscr{P} 11: \max _{\mathbf{R}} & \sigma_{s}^{2}\|\mathbf{R} \mathbf{h}\|^{2}+\sigma_{r}^{2}\|\mathbf{R}\|^{2} \\
\text { s.t. } & \sigma_{s}^{2}\left|\mathbf{g}_{m}^{H} \mathbf{R h}\right|^{2}+\sigma_{r}^{2}\left\|\mathbf{g}_{m}^{H} \mathbf{R}\right\|^{2} \leq I_{m}, \\
& \forall \Delta \mathbf{g}_{m} \in \mathscr{G}_{m}, \quad \forall m .
\end{array}
$$

Denote the optimal objective function value of problem $\mathscr{P} 11$ as $\bar{P}_{r}$. Then, when $P_{r}<\bar{P}_{r}$, increasing $P_{r}$ expands the feasible region of problem $\mathscr{P} 3 ; \gamma_{\text {Pro }}$ as well as the capacity improvement could become larger. On the other hand, when $P_{r} \geq \bar{P}_{r}$, the feasible region of both problem $\mathscr{P} 3$ and problem $\mathscr{P} 4$ keeps invariant as $P_{r}$ increases, indicating that both $\gamma_{\text {Pro }}$ and $\gamma_{\text {Sub }}$ keep almost constant, which leads the capacity improvement to also keep invariant.

\section{Conclusion}

An improved robust relay beamforming design for multiantenna nonregenerative CRN is developed in this paper. The objective is to maximize the worst-case achievable channel capacity for SUs subject to worst-case interference constraint at PUs and transmit power constraint at relay. By employing the optimal beamforming matrix with simplified structure, the original robust beamforming problem is converted into an equivalent problem. Then, an optimal or near-optimal solution of the original problem can be obtained by solving the equivalent one. Analysis and simulation results show that the proposed method can achieve higher capacity for SUs and require lower computational complexity than suboptimal scheme. 


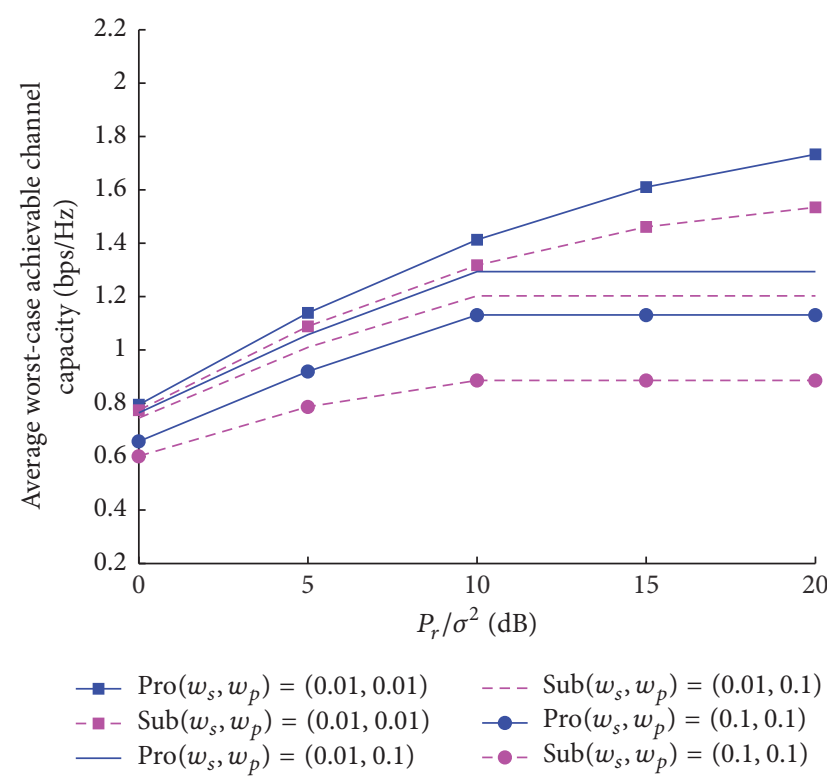

(a)

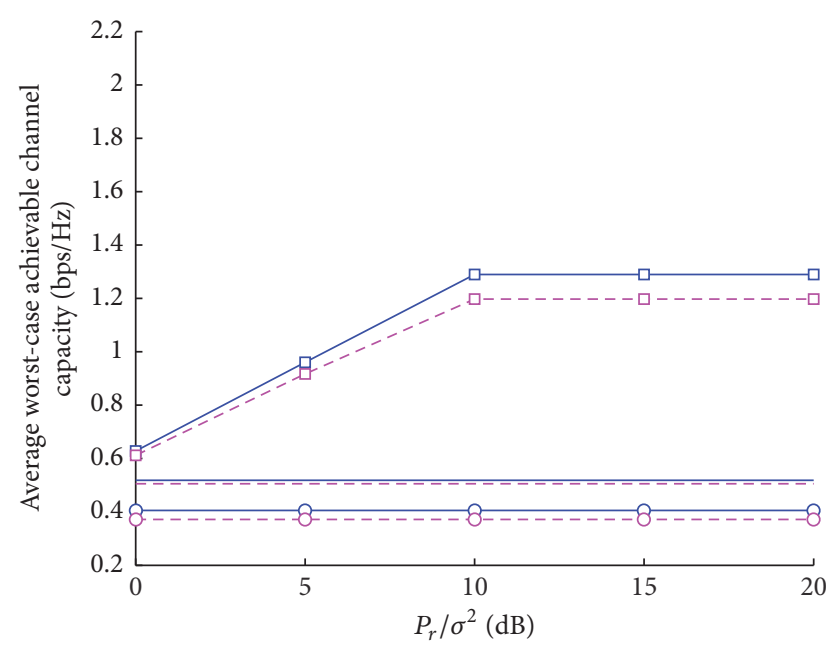

$\begin{array}{ll}\square-\operatorname{Pro}\left(w_{s}, w_{p}\right)=(0.01,0.01) & --\operatorname{Sub}\left(w_{s}, w_{p}\right)=(0.01,0.1) \\ -\square-\operatorname{Sub}\left(w_{s}, w_{p}\right)=(0.01,0.01) & -0-\operatorname{Pro}\left(w_{s}, w_{p}\right)=(0.1,0.1) \\ \square \operatorname{Pro}\left(w_{s}, w_{p}\right)=(0.01,0.1) & -0-\operatorname{Sub}\left(w_{s}, w_{p}\right)=(0.1,0.1)\end{array}$

(b)

FIGURE 4: Average worst-case achievable channel capacity versus relay transmit power budget $P_{r} / \sigma^{2}$. (a) $I / \sigma^{2}=0 \mathrm{~dB}$; (b) $I / \sigma^{2}=-10 \mathrm{~dB}$.

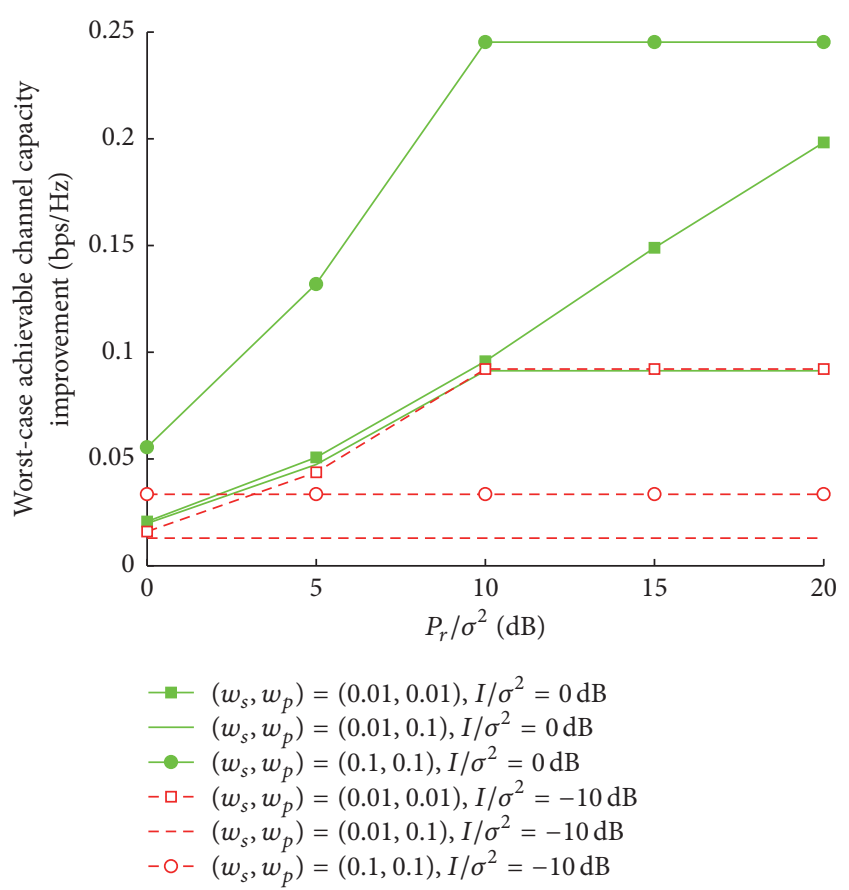

FIgURE 5: Average worst-case achievable channel capacity improvement versus relay transmit power budget $P_{r} / \sigma^{2}$.

\section{Appendix}

\section{A. Proof of Lemma 1}

Proof. Using $\|\mathbf{A}\|^{2}=\operatorname{tr}\left(\mathbf{A} \mathbf{A}^{H}\right)$ and $\mathbf{h}^{H}\left(\mathbf{h}^{\perp}\right)=\mathbf{0}$, we can get

$$
\begin{gathered}
\sigma_{s}^{2}\left\|\left(\mathbf{w h}^{H}\right) \mathbf{h}\right\|^{2}+\sigma_{r}^{2}\left\|\left(\mathbf{w h}^{H}\right)\right\|^{2} \\
\leq \sigma_{s}^{2}\left\|\left(\mathbf{w h}^{H}\right) \mathbf{h}\right\|^{2}
\end{gathered}
$$

$$
\begin{gathered}
+\sigma_{r}^{2}\left(\left\|\left(\mathbf{w} \mathbf{h}^{H}\right)\right\|^{2}+\left\|\mathbf{T}\left(\mathbf{h}^{\perp}\right)^{H}\right\|^{2}\right) \\
=\sigma_{s}^{2}\left\|\mathbf{R}_{o} \mathbf{h}\right\|^{2}+\sigma_{r}^{2}\left\|\mathbf{R}_{o}\right\|^{2} \leq P_{r}, \\
\sigma_{s}^{2}\left|\mathbf{g}_{m}^{H}\left(\mathbf{w} \mathbf{h}^{H}\right) \mathbf{h}\right|^{2}+\sigma_{r}^{2}\left\|\mathbf{g}_{m}^{H}\left(\mathbf{w h}^{H}\right)\right\|^{2} \\
\leq \sigma_{s}^{2}\left|\mathbf{g}_{m}^{H}\left(\mathbf{w h}^{H}\right) \mathbf{h}\right|^{2} \\
+\sigma_{r}^{2}\left(\left\|\mathbf{g}_{m}^{H}\left(\mathbf{w h}{ }^{H}\right)\right\|^{2}+\left\|\mathbf{g}_{m}^{H} \mathbf{T}\left(\mathbf{h}^{\perp}\right)^{H}\right\|^{2}\right) \\
=\sigma_{s}^{2}\left|\mathbf{g}_{m}^{H} \mathbf{R}_{o} \mathbf{h}\right|^{2}+\sigma_{r}^{2}\left\|\mathbf{g}_{m}^{H} \mathbf{R}_{o}\right\|^{2} \leq I_{m}, \\
\quad \forall \Delta \mathbf{g}_{m} \in \mathscr{G}_{m}, \quad \forall m .
\end{gathered}
$$

Inequalities (A.1) imply that $\mathbf{w h}^{H}$ is a feasible solution of problem $\mathscr{P} 3$. Since $\mathbf{R}_{o}$ is the optimal solution of problem $\mathscr{P} 3$, we can obtain

$$
\min _{\Delta \mathbf{g} \in \mathscr{G}} \frac{\sigma_{s}^{2}\left|\mathbf{g}^{H}\left(\mathbf{w h}^{H}\right) \mathbf{h}\right|^{2}}{\sigma_{r}^{2}\left\|\mathbf{g}^{H}\left(\mathbf{w h}^{H}\right)\right\|^{2}+\sigma_{d}^{2}} \leq \min _{\Delta \mathbf{g} \in \mathscr{G}} \frac{\sigma_{s}^{2}\left|\mathbf{g}^{H} \mathbf{R}_{o} \mathbf{h}\right|^{2}}{\sigma_{r}^{2}\left\|\mathbf{g}^{H} \mathbf{R}_{o}\right\|^{2}+\sigma_{d}^{2}} .
$$

On the other hand, we have

$$
\begin{aligned}
& \min _{\Delta \mathbf{g} \in \mathscr{G}} \frac{\sigma_{s}^{2}\left|\mathbf{g}^{H} \mathbf{R}_{o} \mathbf{h}\right|^{2}}{\sigma_{r}^{2}\left\|\mathbf{g}^{H} \mathbf{R}_{o}\right\|^{2}+\sigma_{d}^{2}} \\
& \quad \min _{\Delta \mathbf{g} \in \mathscr{G}} \frac{\sigma_{s}^{2}\left|\mathbf{g}^{H}\left(\mathbf{w} \mathbf{h}^{H}\right) \mathbf{h}\right|^{2}}{\sigma_{r}^{2}\left(\left\|\mathbf{g}^{H}\left(\mathbf{w h}^{H}\right)\right\|^{2}+\left\|\mathbf{g}^{H} \mathbf{T}\left(\mathbf{h}^{\perp}\right)^{H}\right\|^{2}\right)+\sigma_{d}^{2}} \\
& \leq \min _{\Delta \mathbf{g} \in \mathscr{G}} \frac{\sigma_{s}^{2}\left|\mathbf{g}^{H}\left(\mathbf{w h}^{H}\right) \mathbf{h}\right|^{2}}{\sigma_{r}^{2}\left\|\mathbf{g}^{H}\left(\mathbf{w h} \mathbf{h}^{H}\right)\right\|^{2}+\sigma_{d}^{2}} .
\end{aligned}
$$


Combining (A.2) and (A.3), we can obtain

$$
\min _{\Delta \mathbf{g} \in \mathscr{G}} \frac{\sigma_{s}^{2}\left|\mathbf{g}^{H}\left(\mathbf{w} \mathbf{h}^{H}\right) \mathbf{h}\right|^{2}}{\sigma_{r}^{2}\left\|\mathbf{g}^{H}\left(\mathbf{w h} \mathbf{h}^{H}\right)\right\|^{2}+\sigma_{d}^{2}}=\min _{\Delta \mathbf{g} \in \mathscr{G}} \frac{\sigma_{s}^{2}\left|\mathbf{g}^{H} \mathbf{R} \mathbf{g}_{o}\right|^{2}}{\sigma_{r}^{2}\left\|\mathbf{g}^{H} \mathbf{R}_{o}\right\|^{2}+\sigma_{d}^{2}} .
$$

Therefore, $\mathbf{w h}^{H}$ is also the optimal solution of problem $\mathscr{P} 3$.

\section{B. Proof of Lemma 2}

Proof. Assume the optimal solution of problem $\mathscr{P} 8$ is $\mathbf{W}_{o}$; we can verify that $(1-\delta / 2) \mathbf{W}_{o}$ is a strictly feasible solution of problem $\mathscr{P} 9$ since

$$
\begin{gathered}
\mathbf{g}^{H}\left(1-\frac{\delta}{2}\right) \mathbf{W}_{o} \mathbf{g}>(1-\delta) \tau_{o}, \quad \forall \Delta \mathbf{g} \in \mathscr{G}, \\
\mathbf{g}_{m}^{H}\left(1-\frac{\delta}{2}\right) \mathbf{W}_{o} \mathbf{g}_{m}<\alpha I_{m}, \quad \forall \Delta \mathbf{g}_{m} \in \mathscr{G}_{m}, \quad \forall m .
\end{gathered}
$$

always holds. Therefore, the problem $\mathscr{P} 9$ satisfies Slater's constraint qualification condition. Thus, the strong duality holds and the KKT conditions are the sufficient and necessary conditions for a primal-dual point to be optimal [23].

Define

$$
\begin{gathered}
\mathbf{U}_{0} \triangleq\left(\begin{array}{cc}
\widehat{\mathbf{g}}^{H} \mathbf{W} \widehat{\mathbf{g}}-(1-\delta) \tau_{o}-s_{0} & \widehat{\mathbf{g}}^{H} \mathbf{W} \\
\mathbf{W} \widehat{\mathbf{g}} & \mathbf{W}+s_{0} \mathbf{Q}
\end{array}\right), \\
\mathbf{U}_{m} \triangleq\left(\begin{array}{cc}
-\widehat{\mathbf{g}}_{m}^{H} \mathbf{W} \widehat{\mathbf{g}}_{m}+\alpha I_{m}-s_{m} & -\widehat{\mathbf{g}}_{m}^{H} \mathbf{W} \\
-\mathbf{W} \widehat{\mathbf{g}}_{m} & -\mathbf{W}+s_{m} \mathbf{Q}_{m}
\end{array}\right) .
\end{gathered}
$$

The Lagrangian function of problem $\mathscr{P} 9$ can be written as

$$
\begin{aligned}
\mathfrak{Q}(\mathscr{X})= & \operatorname{tr}(\mathbf{W})-\operatorname{tr}\left(\mathbf{U}_{0} \mathbf{V}_{0}\right)-\sum_{m=1}^{M} \operatorname{tr}\left(\mathbf{U}_{m} \mathbf{V}_{m}\right) \\
& -\operatorname{tr}(\mathbf{W Z})-\eta_{0} s_{0}-\sum_{m=1}^{M} \eta_{m} s_{m},
\end{aligned}
$$

where $\mathscr{X}=\{\mathbf{W}, \mathbf{Z}, \mathbf{V}, \boldsymbol{\eta}, \mathbf{s}\}$ consists of all the primal and dual variables with $\mathbf{W} \geq \mathbf{0}, \mathbf{Z} \geq \mathbf{0}, \mathbf{V}=\left\{\mathbf{V}_{m} \geq \mathbf{0}\right\}_{m=0}^{M}, \boldsymbol{\eta}=\left\{\eta_{m} \geq\right.$ $0\}_{m=0}^{M}$, and $\mathbf{s}=\left\{s_{m} \geq 0\right\}_{m=0}^{M}$.

The KKT conditions relevant to the proof are given by

$$
\begin{aligned}
\frac{\partial \mathfrak{Q}(\mathscr{X})}{\partial \mathbf{W}} & =\mathbf{0}, \\
\mathbf{U}_{0} \mathbf{V}_{0} & =\mathbf{0}, \\
\mathbf{W Z} & =\mathbf{0} .
\end{aligned}
$$

Substituting (B.5) into (B.6a), we have

$$
\begin{aligned}
& \frac{\partial \mathfrak{Q}(\mathscr{X})}{\partial \mathbf{W}}=\mathbf{I}-(\widehat{\mathbf{g}} \mathbf{I}) \mathbf{V}_{0}(\widehat{\mathbf{g}} \mathbf{I})^{H} \\
& +\sum_{m=1}^{M}\left(\begin{array}{ll}
\widehat{\mathbf{g}}_{m} & \mathbf{I}
\end{array}\right) \mathbf{V}_{m}\left(\begin{array}{ll}
\widehat{\mathbf{g}}_{m} & \mathbf{I}
\end{array}\right)^{H}-\mathbf{Z}=\mathbf{0}
\end{aligned}
$$

From (B.7), we can get

$$
\begin{aligned}
& \left(\begin{array}{ll}
\widehat{\mathbf{g}} & \mathbf{I}
\end{array}\right) \mathbf{V}_{0}(\widehat{\widehat{g}} \mathbf{I})^{H}+\mathbf{Z}=\mathbf{I} \\
& +\sum_{m=1}^{M}\left(\begin{array}{ll}
\widehat{\mathbf{g}}_{m} & \mathbf{I}
\end{array}\right) \mathbf{V}_{m}\left(\begin{array}{ll}
\widehat{\mathbf{g}}_{m} & \mathbf{I}
\end{array}\right)^{H} .
\end{aligned}
$$

Multiplying both sides of (B.8) by $\mathbf{W}$ and using (B.6c), we can obtain

$$
\begin{aligned}
& \mathbf{W}(\widehat{\widehat{\mathbf{g}}} \mathbf{I}) \mathbf{V}_{0}\left(\begin{array}{ll}
\widehat{\mathbf{g}} & \mathbf{I}
\end{array}\right)^{H} \\
& =\mathbf{W}\left[\mathbf{I}+\sum_{m=1}^{M}\left(\begin{array}{ll}
\widehat{\mathbf{g}}_{m} & \mathbf{I}
\end{array}\right) \mathbf{V}_{m}\left(\begin{array}{ll}
\widehat{\mathbf{g}}_{m} & \mathbf{I}
\end{array}\right)^{H}\right] .
\end{aligned}
$$

Premultiplying and postmultiplying equation (B.6b) by $\left(\begin{array}{ll}\mathbf{0} & \mathrm{I}\end{array}\right)$ and $(\widehat{\mathbf{g}} \mathbf{I})^{H}$, respectively, we have

$$
\left(\begin{array}{ll}
\mathbf{0} & \mathbf{I}
\end{array}\right) \mathbf{U}_{0} \mathbf{V}_{0}\left(\begin{array}{ll}
\widehat{\mathbf{g}} & \mathbf{I}
\end{array}\right)^{H}=\mathbf{0} .
$$

Substituting (B.3) into (B.10) will yield

$$
\begin{aligned}
& \mathbf{0}=\left(\begin{array}{ll}
\mathbf{0} & \mathbf{I}
\end{array}\right)\left(\begin{array}{ll}
\widehat{\mathbf{g}} & \mathbf{I}
\end{array}\right)^{H} \mathbf{W}\left(\begin{array}{ll}
\widehat{\mathbf{g}} & \mathbf{I}
\end{array}\right) \mathbf{V}_{0}\left(\begin{array}{ll}
\widehat{\mathbf{g}} & \mathbf{I}
\end{array}\right)^{H} \\
& -\left(\begin{array}{ll}
\mathbf{0} & \mathbf{I}
\end{array}\right)\left(\begin{array}{cc}
-(1-\delta) \tau_{o}-s_{0} & \mathbf{0}^{H} \\
\mathbf{0} & \mathbf{0}
\end{array}\right) \mathbf{V}_{0}\left(\begin{array}{ll}
\widehat{\mathbf{g}} & \mathbf{I}
\end{array}\right)^{H} \\
& +\left(\begin{array}{ll}
\mathbf{0} & \mathbf{I}
\end{array}\right)\left(\begin{array}{cc}
0 & \mathbf{0}^{H} \\
\mathbf{0} & s_{0} \mathbf{Q}
\end{array}\right) \mathbf{V}_{0}\left(\begin{array}{ll}
\widehat{\mathbf{g}} & \mathbf{I}
\end{array}\right)^{H} \text {. }
\end{aligned}
$$

From (B.11), we can obtain

$$
\mathbf{W}\left(\begin{array}{ll}
\widehat{\mathbf{g}} & \mathbf{I}
\end{array}\right) \mathbf{V}_{0}\left(\begin{array}{ll}
\widehat{\mathbf{g}} & \mathbf{I}
\end{array}\right)^{H}+s_{0} \mathbf{Q}\left(\begin{array}{ll}
\mathbf{0} & \mathbf{I}
\end{array}\right) \mathbf{V}_{0}\left(\begin{array}{ll}
\widehat{\mathbf{g}} & \mathbf{I}
\end{array}\right)^{H}=\mathbf{0} .
$$

Adding $s_{0} \mathbf{Q}\left(\begin{array}{ll}\widehat{\mathbf{g}} & \mathbf{0}\end{array}\right) \mathbf{V}_{0}\left(\begin{array}{ll}\widehat{\mathbf{g}} & \mathbf{I}\end{array}\right)^{H}$ to both sides of (B.12), we can get

$$
\begin{aligned}
& \left(\mathbf{W}+s_{0} \mathbf{Q}\right)(\widehat{\mathbf{g}} \mathbf{I}) \mathbf{V}_{0}(\widehat{\mathbf{g}} \mathbf{I})^{H} \\
& =s_{0} \mathbf{Q}\left(\begin{array}{ll}
\widehat{\mathbf{g}} & \mathbf{0}
\end{array}\right) \mathbf{V}_{0}\left(\begin{array}{ll}
\widehat{\mathbf{g}} & \mathbf{I}
\end{array}\right)^{H} \text {. }
\end{aligned}
$$

If $s_{0}=0$, from (B.13), we have

$$
\mathbf{W}\left(\begin{array}{ll}
\widehat{\mathbf{g}} & \mathbf{I}
\end{array}\right) \mathbf{V}_{0}\left(\begin{array}{ll}
\widehat{\mathbf{g}} & \mathbf{I}
\end{array}\right)^{H}=\mathbf{0} .
$$

Combining (B.9) and (B.14), we can obtain

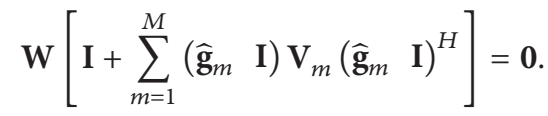

Since $\mathbf{I}+\sum_{m=1}^{M}\left(\widehat{\mathbf{g}}_{m} \mathbf{I}\right) \mathbf{V}_{m}\left(\widehat{\mathbf{g}}_{m} \mathbf{I}\right)^{H}>\mathbf{0}$ always holds, from (B.15), we have $\mathbf{W}=\mathbf{0}$. Then, substituting $\mathbf{W}=\mathbf{0}$ into (B.3), we can get

$$
\mathbf{U}_{0}=\left(\begin{array}{cc}
-(1-\delta) \tau_{o}-s_{0} & \mathbf{0}^{H} \\
\mathbf{0} & s_{0} \mathbf{Q}
\end{array}\right)
$$


However, $\mathbf{U}_{0}$ cannot be semidefinite positive matrix, which violates the assumption that the SDP problem $\mathscr{P} 9$ is feasible. Therefore, $s_{0}$ and $\mathbf{W}$ should satisfy $s_{0}>0$ and $\mathbf{W} \neq \mathbf{0}$, respectively.

Using $\mathbf{W} \succeq \mathbf{0}, \mathbf{Q}>\mathbf{0}$, and $s_{0}>0$, we have

$$
\mathbf{W}+s_{0} \mathbf{Q}>\mathbf{0} .
$$

Because $\mathbf{W}+s_{0} \mathbf{Q}$ is full rank, we can obtain

$$
\begin{aligned}
& \operatorname{rank}\left(\left(\begin{array}{ll}
\widehat{\mathbf{g}} & \mathbf{I}
\end{array}\right) \mathbf{V}_{0}(\widehat{\widehat{\mathbf{g}}} \mathbf{I})^{H}\right) \\
& =\operatorname{rank}\left(\left(\mathbf{W}+s_{0} \mathbf{Q}\right)(\widehat{\mathbf{g}} \mathbf{I}) \mathbf{V}_{0}(\widehat{\mathbf{g}} \mathbf{I})^{H}\right) \text {. }
\end{aligned}
$$

Substituting (B.13) into (B.18) will yield

$$
\begin{aligned}
& \operatorname{rank}\left(\left(\begin{array}{ll}
\widehat{\mathbf{g}} & \mathbf{I}
\end{array}\right) \mathbf{V}_{0}\left(\begin{array}{ll}
\widehat{\mathbf{g}} & \mathbf{I}
\end{array}\right)^{H}\right) \\
& \quad=\operatorname{rank}\left(s_{0} \mathbf{Q}\left(\begin{array}{ll}
\widehat{\mathbf{g}} & \mathbf{0}
\end{array}\right) \mathbf{V}_{0}\left(\begin{array}{ll}
\widehat{\mathbf{g}} & \mathbf{I}
\end{array}\right)^{H}\right) \leq \operatorname{rank}\left(\left(\begin{array}{ll}
\widehat{\mathbf{g}} & \mathbf{0}
\end{array}\right)\right) \\
& \quad=1 .
\end{aligned}
$$

The last inequality is from a basic rank inequality for the product of two matrices [26].

Because $\mathbf{I}+\sum_{m=1}^{M}\left(\widehat{\mathbf{g}}_{m} \mathbf{I}\right) \mathbf{V}_{m}\left(\widehat{\mathbf{g}}_{m} \mathbf{I}\right)^{H}$ is full rank, we have

$\operatorname{rank}(\mathbf{W})$

$$
\left.=\operatorname{rank}\left(\mathbf{W}\left[\begin{array}{ll}
\mathbf{I}+\sum_{m=1}^{M}\left(\begin{array}{ll}
\widehat{\mathbf{g}}_{m} & \mathbf{I}
\end{array}\right) \mathbf{V}_{m}\left(\widehat{\mathbf{g}}_{m}\right. & \mathbf{I}
\end{array}\right)^{H}\right]\right) .
$$

Substituting (B.9) into (B.20), we can get

$$
\begin{aligned}
\operatorname{rank}(\mathbf{W}) & =\operatorname{rank}\left(\mathbf{W}\left(\begin{array}{ll}
\widehat{\mathbf{g}} \mathbf{I}
\end{array}\right) \mathbf{V}_{0}\left(\begin{array}{ll}
\widehat{\mathbf{g}} & \mathbf{I}
\end{array}\right)^{H}\right) \\
& \leq \operatorname{rank}\left(\left(\begin{array}{ll}
\widehat{\mathbf{g}} & \mathbf{I}
\end{array}\right) \mathbf{V}_{0}\left(\begin{array}{ll}
\widehat{\mathbf{g}} & \mathbf{I}
\end{array}\right)^{H}\right)
\end{aligned}
$$

Combing (B.19) and (B.21), we can conclude that

$$
\operatorname{rank}(\mathbf{W}) \leq 1 \text {. }
$$

On the other hand, since $\mathbf{W} \neq \mathbf{0}$ holds, we have $\operatorname{rank}(\mathbf{W})=1$; that is, the optimal solution of problem $\mathscr{P} 9$ is rank-one.

\section{Competing Interests}

The authors declare that there is no conflict of interests regarding the publication of this paper.

\section{Acknowledgments}

This paper is supported by the Natural Science Foundation of China (no. 61401278).

\section{References}

[1] S. Haykin, "Cognitive radio: brain-empowered wireless communications," IEEE Journal on Selected Areas in Communications, vol. 23, no. 2, pp. 201-220, 2005.
[2] A. Ghasemi and E. S. Sousa, "Fundamental limits of spectrumsharing in fading environments," IEEE Transactions on Wireless Communications, vol. 6, no. 2, pp. 649-658, 2007.

[3] A. Goldsmith, S. A. Jafar, I. Maric, and S. Srinivasa, "Breaking spectrum gridlock with cognitive radios: an information theoretic perspective," Proceedings of the IEEE, vol. 97, no. 5, pp. 894-914, 2009.

[4] J. Lee, H. Wang, J. G. Andrews, and D. Hong, "Outage probability of cognitive relay networks with interference constraints," IEEE Transactions on Wireless Communications, vol. 10, no. 2, pp. 390-395, 2011.

[5] L. Luo, P. Zhang, G. Zhang, and J. Qin, "Outage performance for cognitive relay networks with underlay spectrum sharing," IEEE Communications Letters, vol. 15, no. 7, pp. 710-712, 2011.

[6] M. Xia and S. Aïssa, "Cooperative AF relaying in spectrumsharing systems: performance analysis under average interference power constraints and Nakagami-m fading," IEEE Transactions on Communications, vol. 60, no. 6, pp. 1523-1533, 2012.

[7] R. Pabst, B. Walke, D. Schultz et al., "Relay-based deployment concepts for wireless and mobile broadband radio," IEEE Communications Magazine, vol. 42, no. 9, pp. 80-89, 2004.

[8] A. Sendonaris, E. Erkip, and B. Aazhang, "User cooperation diversity. part I. system description," IEEE Transactions on Communications, vol. 51, no. 11, pp. 1927-1938, 2003.

[9] J. N. Laneman, D. N. C. Tse, and G. W. Wornell, "Cooperative diversity in wireless networks: efficient protocols and outage behavior," IEEE Transactions on Information Theory, vol. 50, no. 12, pp. 3062-3080, 2004.

[10] Q. Li, L. Luo, and J. Qin, "Optimal relay precoder for nonregenerative MIMO cognitive relay systems with underlay spectrum sharing," Electronics Letters, vol. 48, no. 5, pp. 295297, 2012.

[11] K. R. Budhathoki, M. Maleki, and H. R. Bahrami, "Precoder design for non-regenerative MIMO relay cognitive radio systems," in Proceedings of the IEEE 78th Vehicular Technology Conference (VTC Fall '13), Las Vegas, Nev, USA, September 2013.

[12] R. Feng, Q. Li, Q. Zhang, and J. Qin, "Optimal beamforming in spectrum sharing cognitive non-regenerative multi-relay networks," Electronics Letters, vol. 49, no. 17, pp. 1102-1104, 2013.

[13] P. Ubaidulla and S. Aissa, "Robust distributed cognitive relay beamforming," in Proceedings of the IEEE 75th Vehicular Technology Conference (VTC Spring '12), Yokohama, Japan, May 2012.

[14] S. Mohammadkhani, M. H. Kahaei, and S. M. Razavizadeh, "Robust beamforming and power allocation in cognitive radio relay networks with imperfect channel state information," IET Communications, vol. 8, no. 9, pp. 1560-1569, 2014.

[15] S. Singh, P. D. Teal, P. A. Dmochowski, and A. J. Coulson, "Robust cooperative relay beamforming for cognitive radio networks," in Proceedings of the Australian Communications Theory Workshop (AusCTW '16), pp. 89-94, Melbourne, Australia, January 2016.

[16] S. Ma, Z.-L. Dong, J. P. Ma, and D. C. Sun, "Robust relay beamforming in cognitive two-way relay networks," IEEE Communications Letters, vol. 17, no. 10, pp. 2000-2003, 2013.

[17] Q. Li, Q. Zhang, and J. Qin, “Robust beamforming for cognitive multi-antenna relay networks with bounded channel uncertainties," IEEE Transactions on Communications, vol. 62, no. 2, pp. 478-487, 2014. 
[18] S. Wen, X. Zhu, Y. Lin, Z. Lin, X. Zhang, and D. Yang, "Achievable transmission capacity of relay-assisted device-todevice (D2D) communication underlay cellular networks," in Proceedings of the IEEE 78th Vehicular Technology Conference (VTC Fall '13), September 2013.

[19] J. Li, J. B. Song, and Z. Han, "Network connectivity optimization for device-to-device wireless system with femtocells," IEEE Transactions on Vehicular Technology, vol. 62, no. 7, pp. 30983109, 2013.

[20] G. Zheng, K.-K. Wong, and B. Ottersten, "Robust cognitive beamforming with bounded channel uncertainties," IEEE Transactions on Signal Processing, vol. 57, no. 12, pp. 4871-4881, 2009.

[21] L. Zhang, Y.-C. Liang, Y. Xin, and H. V. Poor, "Robust cognitive beamforming with partial channel state information," IEEE Transactions on Wireless Communications, vol. 8, no. 8, pp. 4143-4153, 2009.

[22] A. Beck and Y. C. Eldar, "Strong duality in nonconvex quadratic optimization with two quadratic constraints," SIAM Journal on Optimization, vol. 17, no. 3, pp. 844-860, 2006.

[23] S. Boyd and L. Vandenberghe, Convex Optimization, Cambridge University Press, Cambridge, UK, 2004.

[24] I. Polik and T. Terlaky, "Interior point methods for nonlinear optimization," in Nonlinear Optimization, G. Di Pillo and F. Schoen, Eds., Springer, 1st edition, 2010.

[25] M. Grant and S. Boyd, CVX: Matlab Software for Disciplined Convex Programming (Version 2.1, Build 1110), 2015, http://cvxr .com/cvx/.

[26] G. H. Golub and C. F. Van Loan, Matrix Computations, JHU Press, Baltimore, Md, USA, 2012. 

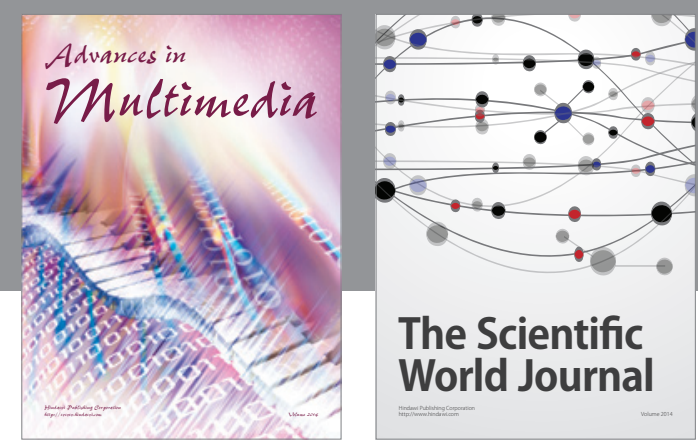

The Scientific World Journal
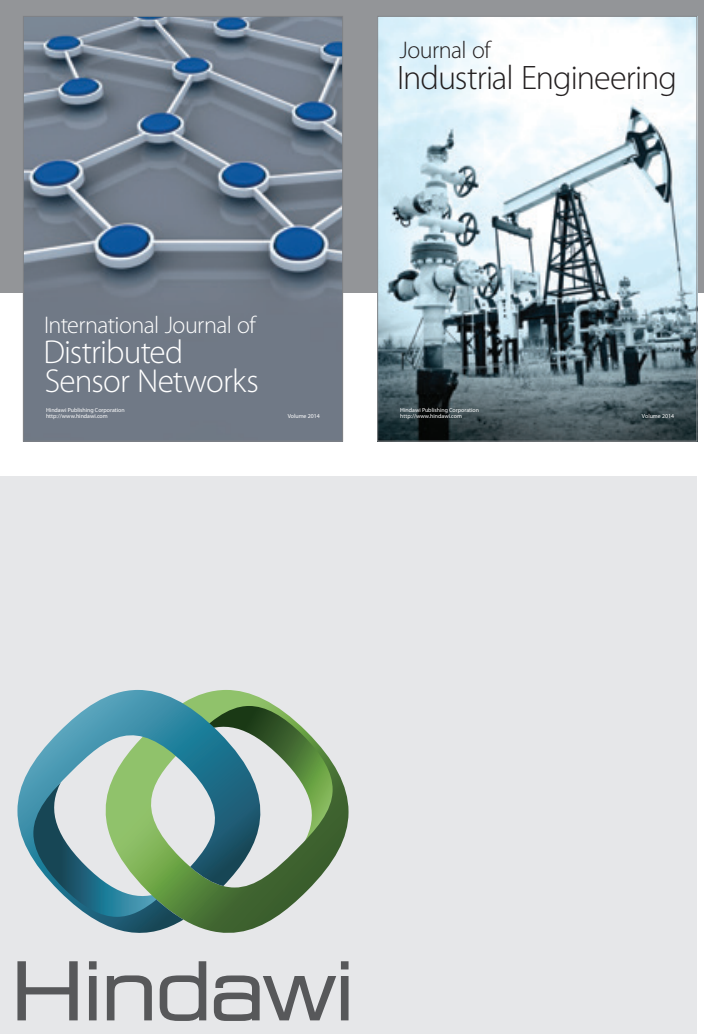

Submit your manuscripts at

https://www.hindawi.com

\section{Computer Networks} and Communications
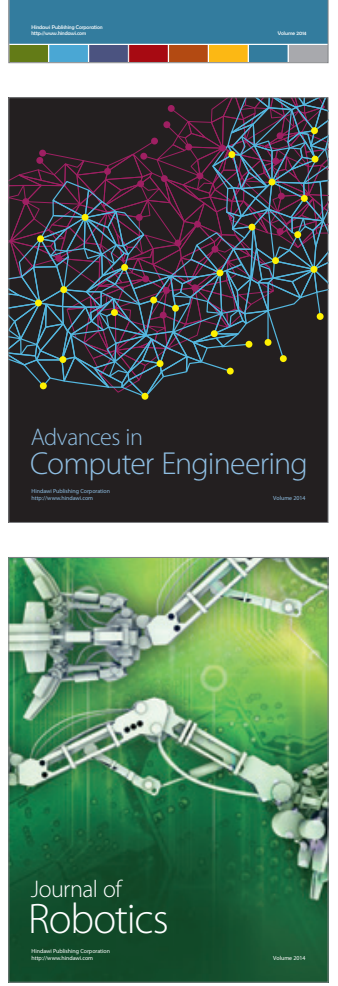
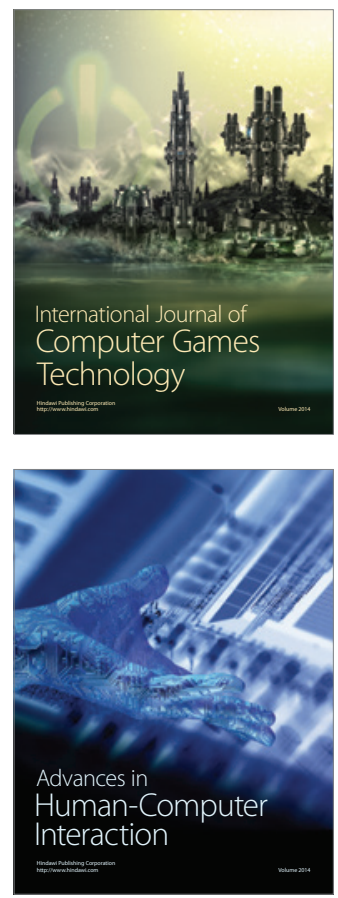
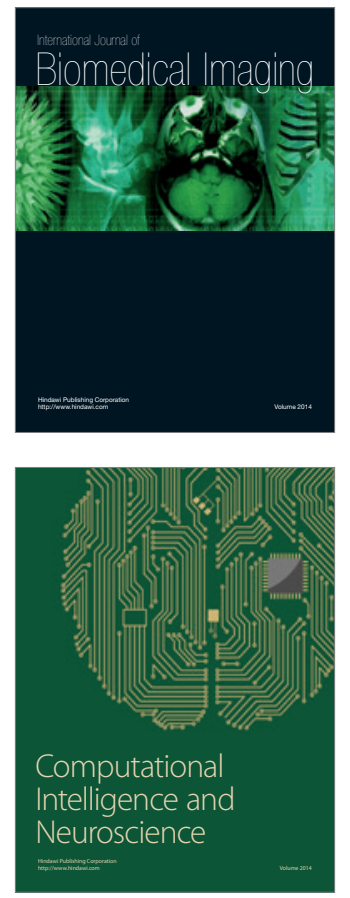
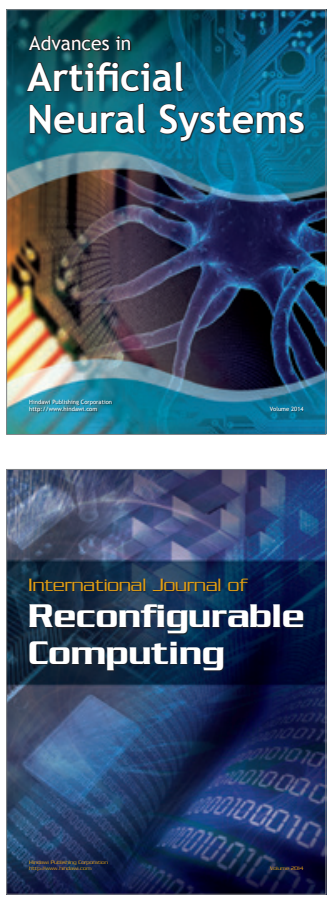
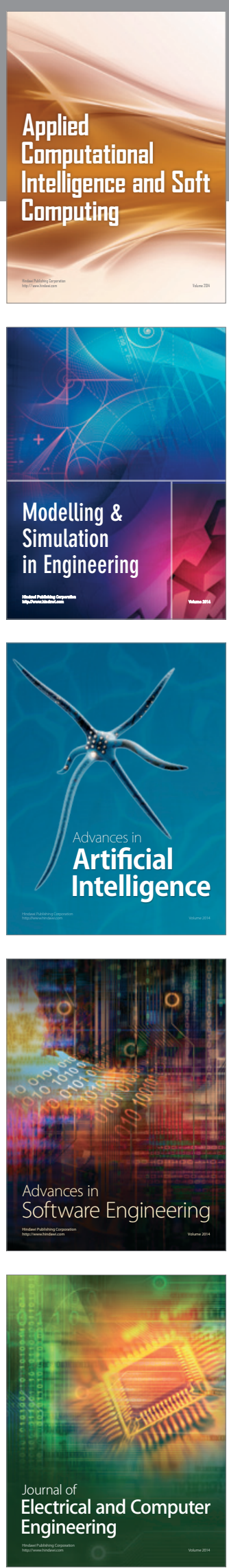